\title{
Patients' perception of visual impairment in glaucoma: a pilot study
}

Patricia Nelson, Peter Aspinall, Colm O’Brien

\begin{abstract}
Backgroundlaims-There is a paucity of useful information on the level of visual disability suffered by glaucoma patients. The aims of this study were to determine and rank the frequency of self reported visual disability in daily tasks performed by glaucoma patients; to examine the interrelation between disabilities using factor analysis; to study the relation between perceived visual difficulty and a measure of the severity of visual field loss; to develop a glaucoma specific subgroup of questions; and examine the validity and reliability of this subgroup of questions.
\end{abstract}

Methods-63 glaucoma patients completed a questionnaire containing 62 questions covering 10 broad aspects of daily life activities using a five point answer scale. Patients were classified into three groups as having mild, moderate, and severe field loss on the basis of the perimetric results. The relation between a measure of the severity of visual field loss and subjective visual disability in the three groups was examined.

Results-Using factor analysis, the most frequently reported problems were grouped into the following four categories: outdoor mobility, glare and lighting conditions and activities demanding functional peripheral vision, household tasks, and personal care. These four factors accounted for $72 \%$ of the variability in the patients' questionnaire responses. With increasing severity of binocular visual field loss there was an increase in the number of self reported visual problems. A loss of confidence in performing some routine daily tasks tended to precede self reported specific visual disabilities. The factor "glare and lighting and activities demanding functional peripheral vision" was found to have a significant relation with a measure of visual field loss and was used to create a glaucoma specific subset of questions. Cronbach's $\alpha$ showed a high degree of reliability and internal consistency $(\alpha=0.96)$ in this glaucoma specific subset of questions. Furthermore, the validity of this new subset of questions was shown to be significant $(r=0.037, p<0.05)$ for the correlation between a measure of the severity of binocular visual field loss and the mean score of the variables used in the glaucoma specific subgroup of questions.

Conclusions-Outcome measures and quality of life issues need to be addressed in glaucoma. This pilot study identified common problems encountered by patients which at the present time are not assessed in routine glaucoma care. It also identified a subgroup of questions that seems to be specific for glaucoma. Further research is required if a significant impact on the quality of life of glaucoma patients is to be achieved.

(Br f Ophthalmol 1999;83:546-552)

In a recent paper by Quigley ${ }^{1}$ it was pointed out that glaucoma is the second leading cause of visual loss in the world, and the estimated number of people suffering from this eye disease worldwide in the year 2000 will be approximately 67 million. About $10 \%$ of those with this disease may suffer from bilateral blindness, while the remaining $90 \%$ have varying degrees of visual impairment and disability. Loss of vision in glaucoma is irreversible; those who have glaucoma have to live with their disease and cope with its consequences.

Outcome assessment has become increasingly important as a critical measure for treatment and management of medical conditions, and in this respect Zimmerman et $a l^{2}$ and Lee have recently highlighted this issue in glaucoma. Reduced vision, as shown in many studies, is correlated with perceived difficulties in everyday tasks. ${ }^{4-9}$ Very little is known about the impact of glaucoma on the quality of life of those affected. ${ }^{2}$ For many patients the ability to remain independent is not crucially influenced, but little knowledge is available on the extent and character of visual disability experienced by patients in their daily life. More scientific information is needed on the evaluation of patient capabilities in performing visual tasks and on the correlation of perceived disabilities with psychophysical testing of visual function.

The aims of this study were to identify the most commonly perceived disabilities in the daily life of glaucoma patients by means of a questionnaire, to rank the perceived problems with regard to frequency, to group related visual problems and assess their impact on daily life activities, and to examine the relation between perceived visual difficulties and the severity of visual field loss, looking in particular at those variables which could discriminate between different grades of visual field loss. A pilot questionnaire was developed for the purpose of this study. A further task was to specify glaucoma specific subgroup of questions and to test the validity and reliability of this newly created questionnaire subscale. 


\section{Subjects}

VISUAL DISABILITY QUESTIONNAIRE

Sixty three patients attending the glaucoma review clinic within a 3 month period were enrolled in the study. There were 31 males and 32 females in the sample. The mean age of the sample was 70 years (SD 14 years) ranging from 45 to 90 . Snellen visual acuity varied between $6 / 4$ and $6 / 36$, with the mean value of $6 / 6$. Patients with clinically significant cataract, macular degeneration, or any other ophthalmic condition were excluded from the study. Glaucoma was diagnosed on the basis of glaucomatous disc cupping and reproducible visual field damage in one or both eyes. Eighty per cent of the subjects suffered from primary open angle glaucoma (intraocular pressure $>21 \mathrm{~mm} \mathrm{Hg}$ ), and the $20 \%$ were patients with other types of chronic glaucoma (normal pressure, angle closure, pseudoexfoliative). All 63 patients completed the questionnaire.

RELATING SUBJECTIVE VISUAL DISABILITY TO A MEASURE OF SEVERITY OF BINOCULAR VISUAL FIELD LOSS

Although the questionnaire was anonymous, a subsample of 39 patients spontaneously agreed to give us their names and to get some more information on their visual field loss. Further analysis of the data in relation to the severity of visual field loss was performed on these patients. Patients in this subsample suffered from different degrees of visual field loss. Twenty three men and 16 women were included in this analysis, with a mean age 71 years (SD 10 years) ranging from 45 to 90 . Only patients with Snellen visual acuity less or equal to $6 / 12$ in the better eye were included in the study (mean VA 6/6). Only two patients had vision in the fellow eye worse than $6 / 12$. The central 30 degrees of visual fields (threshold and suprathreshold strategies) were plotted using automated perimetry and the central visual fields were classified (by CO'B) into three groups of severity as mild, moderate, or severe (details in Methods). Using this qualitative subdivision, 10 patients had mild field loss, 15 had moderate damage, and 14 severe visual field loss. The groups were compared for difference in relation to age, sex, or visual acuity using the $\chi^{2}$ test and Kruskal-Wallis ANOVA. No statistically significant difference was found in relation to any of these categories in the three groups of visual field loss.

\section{Methods}

VISUAL DISABILITY QUESTIONNAIRE

A pilot questionnaire was used to record self reported disability in glaucoma patients. The process of developing this questionnaire benefited from previous studies on visual disability in glaucoma ${ }^{1011}$ and other ocular conditions, ${ }^{912-15}$ as well as from the clinical experience of a glaucoma specialist (CO'B).

All patients were interviewed by the same person (PN) before they were given the questionnaire. Note of patients' age, sex, Snellen visual acuity, and diagnosis was made. Patients with clinically significant cataract, macular degeneration, or any other ophthalmic condition were excluded from the study. The task of the interviewer was to make sure that every participant understood the nature of the study and how to answer the questions on a five point scale ranging from "no difficulty at all" to "severe difficulty". It was also made clear that patients had to answer questions in relation to their vision alone. An extra option was given in the questionnaire in case the patient did not carry out a particular task for other than visual reasons. After the short interview, patients were asked to complete the questionnaire themselves during the time they were waiting in the clinic. The questions were formulated in plain English and easy to understand.

The questionnaire comprised a total of 62 questions. These covered 47 different activities of daily living (ADL) in 10 main areas of daily life: mobility indoors and outdoors, housework, reading, watching television (TV), social life, leisure activities, travelling, ability to enjoy scenery, and driving.

Confidence in performing routine daily tasks As the questionnaire took 20-30 minutes to complete, only a subgroup of 35 patients were administered a further group of 19 questions dealing with the subject of patients' confidence rather than disability in performing certain tasks. These patients were asked how confident they felt to carry out daily tasks such as cooking, crossing the road, walking on steps, etc. The purpose was to find out whether patients experience increased anxiety and lack of confidence in their daily life resulting from their visual difficulties.

RELATING SUBJECTIVE VISUAL DISABILITY TO A MEASURE OF SEVERITY OF BINOCULAR VISUAL FIELD LOSS

The central 30 degrees of visual fields (threshold and suprathreshold strategies) were plotted with the Humphrey visual field analyser (Humphrey Instruments, Inc; Allergan Humphrey, San Leandro, CA, USA) or the Medmont automated perimeter (Medmont Pty Ltd, Melbourne, Australia). The central visual fields were classified (by CO'B) into three groups of severity as mild (unilateral loss with less than half of the visual field lost), moderate (unilateral loss with more than half of the visual field lost or bilateral loss with less than half of the visual field lost in each eye), or severe (bilateral loss, more than a half of the visual field lost in either eye).

\section{STATISTICAL ANALYSIS}

Visual disability questionnaire

Factor analysis using the Varimax rotation of the principal component analysis provided by SPSS statistical software package (SPSS for Windows; Version 6.0, SPSS, Chicago, IL, USA) was used to process the results of the questionnaire. It is a data reduction technique, in which an initial set of intercorrelations between variables is given a simplified structure by the formation of groups from the initial set. A small number of new groups or patterns emerged called factors which account for most of the variation in patients' responses. 
Table 1 Frequency of self reported difficulties

\begin{tabular}{lll}
\hline & $\begin{array}{l}\text { Patients who failed to answer } \\
\text { or did not perform activity for } \\
\text { non-visual reasons }\end{array}$ & Activities \\
\hline $70 \%$ & $2 \%$ & Glare \\
$54 \%$ & $5 \%$ & Adaptation to different levels of lighting \\
$49 \%$ & $2 \%$ & Walking on steps or kerbs \\
$43 \%$ & $6 \%$ & Reading newspapers \\
$42 \%$ & $3 \%$ & Shopping \\
$40 \%$ & $30 \%$ & Needlework \\
$36 \%$ & $2 \%$ & Crossing the road \\
$32 \%$ & $2 \%$ & Recognising faces and expressions \\
$26 \%$ & $3 \%$ & Using bus or train \\
$26 \%$ & $4 \%$ & Watching television \\
$25 \%$ & $6 \%$ & Indoor mobility \\
$20 \%$ & $6 \%$ & Visiting friends or restaurants \\
$17 \%$ & $7 \%$ & Housework and cooking \\
$17 \%$ & $5 \%$ & Enjoyment of scenery \\
\hline
\end{tabular}

Relating subjective visual disability to a measure of severity of binocular visual field loss

Using SPSs, a Kruskal-Wallis one way analysis of variance (ANOVA) was performed on the three groups (mild, moderate, and severe field loss) followed by the Mann-Whitney U test for two independent samples. As the direction of significant difference was predicted a priorithat is, with progressing field loss increased visual disability was expected, a one tail test was used for analysis of the data. A probability value of $\mathrm{p}<0.05$ was considered as a critical level for significant results.

\section{Validity and reliability of the glaucoma specific subset of questions}

Those activities which best separated the groups with different levels of visual field loss were used to create a glaucoma specific subset of questions. The validity of this newly created subset of questions was evaluated using Spearman correlation coefficient between the computed average score for those questions and the severity of the visual field loss. Reliability analysis of the subset of glaucoma specific questions was carried out using Cronbach $\alpha$ as a measure of internal consistency (SPSS for Windows; Version 6.0, SPSS, Chicago, IL, USA).

\section{Results}

VISUAL DISABILITY QUESTIONNAIRE

The frequency of reported difficulties is presented in Table 1. A high percentage of glaucoma patients complained about frequent problems with common, everyday activities. Of particular note was the percentage of patients who experienced problems with glare $(70 \%)$ and adaptation to different levels of lighting $(54 \%)$ followed by difficulties when walking on steps or kerbs (49\%), reading activities $(43 \%)$, shopping $(42 \%)$, crossing the road $(36 \%)$, using the bus or train $(26 \%)$, visiting friends and restaurants $(20 \%)$, etc. Most vehicle drivers also complained of increased difficulty with glare when driving towards the sun or oncoming headlights $(72 \%)$. A small number of patients (approximately 10\%) mentioned that because of problems with glare they had to stop driving at night or during winter months. Among other examples of reported visual disability were driving at night time $(52 \%)$, the ability to see the control panel in the car at
Table 2 Frequency of occurrence of the main groups of difficulties experienced by patients suffering from glaucoma

\begin{tabular}{ll}
\hline Factors & Frequencies \\
\hline Glare and lighting & $70 \%$ \\
Outdoor mobility day and night & $32-56 \%$ \\
Household tasks & $17 \%$ \\
Personal care & $8-12 \%$ \\
\hline
\end{tabular}

Frequency of occurrence shows the number of patients experiencing difficulty with the listed groups of activities.

night $(33 \%)$, the ability to see traffic signs during day time (15\%), and reversing (15\%).

Factor analysis

While the frequency of reported difficulties gives information about their occurrence (that is, presence or absence of problems), factor analysis deals with interrelations within the data. It reveals therefore the key groups of questions which underpin the problems reported by the patients.

Factor analysis identified nine new groups of questions (factors). Taken together, the first four factors accounted for most of the variability in patients' responses $(72 \%)$ and are summarised under the following general headings: outdoor mobility/navigation, lighting and glare and activities demanding functional peripheral vision, household tasks, and personal care. The technique simplified the 62 questions in the questionnaire into the four main groups and arranged them in descending order in which they accounted for the variability in patients responses. To test the stability of this structure, a second factor analysis was performed with a smaller set of 18 questions. The activities with high $(r>0.7)$ and moderate $(r>0.5)$ correlations on the first five factors were included in this part of analysis. An identical factor structure was obtained with this secondary analysis.

The frequency of occurrence of difficulties related to these four groups can be found in Table 2. The greatest frequency was observed in the second factor, lighting and glare and activities demanding functional peripheral vision (Table 2).

Questions that correlated best on the four factors are listed in Table 3. For the first factor, highly correlated activities were observed relating to outdoor mobility/ navigation such as walking outside in the street during the day or at night, crossing the road, moving in traffic, and also activities related to judging distances. The second factor (lighting and glare and activities demanding functional peripheral vision) indicated difficulty with disability glare and adaptation to different levels of lighting either indoors or outdoors. Activities demanding functional peripheral vision such as tripping over when walking, bumping into objects, or failing to see people or objects in the periphery also correlated mostly on this factor, even though the correlation was not as strong as for glare disability and lighting. Ability to judge distances correlated evenly on the first two factors. The third factor (household tasks) clearly defined problems with typical household activities indoors and in the garden. Personal care 
Table 3 Factor structure of the data. Intercorrelation coefficients of different daily activities on the first four factors that accounted for most of the variance in the patients' responses

\begin{tabular}{|c|c|c|c|c|}
\hline Daily activities & $\begin{array}{l}\text { Factor } 1 \\
\text { Outdoor } \\
\text { mobility }\end{array}$ & $\begin{array}{l}\text { Factor } 2 \\
\text { Glare and } \\
\text { lighting }\end{array}$ & $\begin{array}{l}\text { Factor } 3 \\
\text { Household } \\
\text { tasks }\end{array}$ & $\begin{array}{l}\text { Factor } 4 \\
\text { Personal } \\
\text { care }\end{array}$ \\
\hline Outdoor mobility in general & 0.70 & - & - & - \\
\hline Crossing the road & 0.77 & - & - & - \\
\hline Seeing moving vehicles & 0.78 & - & - & - \\
\hline Walking outdoors after dark & 0.75 & - & - & - \\
\hline Ability to see outdoors after dark & 0.71 & - & - & - \\
\hline Judging distances & 0.57 & - & - & - \\
\hline Glare in general & - & 0.70 & - & - \\
\hline Adaptation to different levels of lighting & - & 0.75 & - & - \\
\hline Bumping into objects & - & 0.62 & - & - \\
\hline Seeing in periphery & - & 0.63 & - & - \\
\hline Tripping over objects & - & 0.59 & - & - \\
\hline Cooking & - & - & 0.79 & - \\
\hline Housework & - & - & 0.73 & - \\
\hline Gardening & - & - & 0.63 & - \\
\hline Dressing & - & - & - & 0.85 \\
\hline Washing & - & - & - & 0.83 \\
\hline Colour vision & - & - & - & 0.82 \\
\hline
\end{tabular}

tasks like dressing, washing, and bathing correlated on the fourth factor.

RELATING SUBJECTIVE VISUAL DISABILITY TO A MEASURE OF SEVERITY OF BINOCULAR VISUAL FIELD DEFECT

Further analysis of the data was carried out on a subgroup of 39 patients as described above. Kruskal-Wallis ANOVA and Mann-Whitney tests were performed on the three groups of patients with different levels of visual field loss (mild, moderate, and severe) (Table 4).

Using the ANOVA assessment significant differences across all groups were found in patient confidence when going out in the street $(p=0.01)$ and in tripping over when walking $(p=0.04)$. Glare disability when adjusting to high levels of lighting had a borderline probability value $(p=0.055)$ (Table 4$)$. These variables are related to the first and second factor of the factor structure ("outdoor mobility", "glare and lighting and activities demanding functional peripheral vision"). Also, in the responses to the general question on perceived "difficulty in activities of daily living (ADL)" there was a significant difference between the groups $(p=0.04)$.

The ANOVA was followed by MannWhitney $U$ test for two independent samples (Table 4). With increasing severity of binocular visual field loss, the number of significant differences between the groups increased. The only significant difference found between mild and moderate visual field loss groups was in two general questions on "activities of daily living performed in dim lighting" $(p=0.03)$ and" activities of daily living in general"
Table 5 Comparison of the combined group of mild and moderate field loss with the severe binocular visual field loss group

\begin{tabular}{ll}
\hline Activities & $p$ Value \\
\hline Adjusting to high levels of lighting & 0.02 \\
Disability glare in general & 0.02 \\
Tripping over & 0.04 \\
Going from bright to dark room or vice versa & 0.055 \\
\hline
\end{tabular}

$(p=0.04)$. There were no differences in any of the specific daily tasks among these two groups of patients. When comparing the groups with moderate and severe field loss the best predictors were a confidence question on "going out in the street" $(p=0.01)$ and glare disability $(\mathrm{p}=0.02)$. Significant differences were found between groups with mild and severe visual field loss in the questions on performance in ADL in general $(p=0.01)$, tripping over objects (0.01), confidence when going out in the street $(p=0.02)$, and adaptation when going from dark to light room or vice versa $(\mathrm{p}=0.05)$.

Finally, the groups with mild and moderate loss were combined and compared with the group with severe loss (Table 5). The MannWhitney $\mathrm{U}$ test showed significant differences between these two groups in adjusting to bright lighting $(p=0.02)$, a general question on difficulty with glare $(p=0.02)$, and tripping over objects $(p=0.04)$. Adaptation when going from a bright to a dark room or vice versa had a borderline probability value $(p=0.055)$. All these questions were related to the second factor of the factor structure.

VALIDITY AND RELIABILITY OF THE

QUESTIONNAIRE SUBSCALES

Validity

This questionnaire was based on several examples in the literature from a range of ophthalmic conditions including glaucoma. The purpose was to find questions that would show relation with a measure of severity of visual field loss. The validity of the questionnaire could therefore be tested only in relation to possible glaucoma specific subgroup consisting of the questions found to be able to discriminate or contribute to the discrimination between the three groups with varying degrees of visual field loss. The validity of the specific questions was demonstrated in their relation with a measure of visual field loss (Tables 4 and 5). Most of these questions were related to the factor "glare and lighting and activities demanding functional peripheral vision". We also computed the average score (mean value) for

Table 4 Group differences in visual disability questionnaire responses with regard to the severity of binocular visual field loss

\begin{tabular}{|c|c|c|c|c|}
\hline \multirow[b]{2}{*}{ Activities } & \multirow{2}{*}{$\begin{array}{l}\begin{array}{l}\text { Kruskal-Wallis } \\
\text { one way ANOVA }\end{array} \\
\begin{array}{l}\text { All groups } \\
\text { (p value) }\end{array}\end{array}$} & \multicolumn{3}{|c|}{ Mann-Whitney U Test } \\
\hline & & $\begin{array}{l}\text { Mild v moderate } \\
(p \text { value })\end{array}$ & $\begin{array}{l}\text { Moderate v severe } \\
\text { ( } p \text { value })\end{array}$ & $\begin{array}{l}\text { Mild v severe } \\
(p \text { value })\end{array}$ \\
\hline $\mathrm{ADL}$ in general & 0.04 & 0.04 & & 0.01 \\
\hline ADL performed in dim lighting & & 0.03 & & \\
\hline Adjusting to bright lighting & 0.055 & & 0.02 & \\
\hline Tripping over & 0.04 & & & 0.01 \\
\hline Going from bright to dark room or vice versa & & & & 0.05 \\
\hline Confidence in going out in the street & 0.01 & & 0.03 & 0.02 \\
\hline
\end{tabular}

$\mathrm{ADL}=$ activities of daily living. 
Table 6 Comparison of the factor structure reported by Ross et al $l^{10}$ with the factor structure resulting from this study

\begin{tabular}{ll}
\hline Present study & Ross et al \\
\hline Outdoor mobility & Navigation outdoors \\
Disability glare and lighting & Navigation at night \\
Household tasks & Vision when cooking \\
Personal tasks & - Near vision \\
Near vision & \\
\hline
\end{tabular}

these questions and examined its correlation with our measure of severity of binocular visual field loss. The Spearman coefficient was used for evaluation of this relation with the resulting value of $r=0.37(\mathrm{p}<0.05)$.

\section{Reliability}

The Cronbach $\alpha$ showed high internal consistency for all the subscales of the questionnaire as suggested by the factor analysis: outdoor mobility (0.96), disability glare and lighting and activities demanding functional peripheral vision (0.93), household tasks (0.92), personal care (0.97). Internal consistency of a possible glaucoma specific subgroup of questions related to factor "glare and lighting and actions demanding functional peripheral vision" was also found to be high (0.96).

\section{Discussion}

VISUAL DISABILITY QUESTIONNAIRE

This study has identified outdoor mobility, glare and lighting, household tasks, and personal care as the main groups of problems encountered by glaucoma patients. This factor structure confirmed the previous findings of Ross et al (Table 6). ${ }^{10}$ Both the sample in the study of Ross and the sample in this study were comparable in their size and considered the same age group. Both studies examined subjects with varying degrees of the visual field loss and used a similar technique to analyse the data.

The first factor described by Ross as navigation outdoors ${ }^{10}$ is identical with the group of experienced disabilities that correlated on the factor outdoor mobility in our study. Problems with navigation at night ${ }^{10}$ indicating difficulty with adaptation to different levels of lighting were confirmed in this study, in relation to the second factor. The questions related to glare disability and activities demanding functional peripheral vision, like tripping over and bumping into objects or ability to see objects coming from the side also correlated on this factor. Naturally, one would expect a correlation of activities demanding functional peripheral vision on the outdoor mobility factor. This was partially the case as the correlation of these activities was spread across the first two factors, but predominant on the second one. It is difficult to explain at this stage why the relation of these activities with glare problems seems to be stronger than with outdoor mobility difficulties.

Vision when cooking ${ }^{10}$ was one of the activities that correlated on the factor with the general heading of household tasks in this study. Near vision ${ }^{10}$ corresponds with the fifth factor in our study. This factor did not significantly increase the proportion of variance in the patients' responses and therefore was not described in detail. In relation to the use of factor analysis, it is necessary to note that because the sample size in this study was smaller than the usually recommended ratio of 2:1 (number of subjects:number of questions), or 20 times the number of factors, ${ }^{16}$ a secondary analysis was performed to test the stability of the factor structure. A smaller set of 18 questions that correlated strongly $(r>0.7)$ or moderately $(r>0.5)$ on the first five factors were entered into the secondary analysis and an identical factor structure was obtained.

A loss of confidence in performing certain tasks was observed by the glaucoma patients in this study before real problems with visual disability were apparent, an observation also reported by Ross et al. ${ }^{10}$ These difficulties were particularly related to outdoor mobility (going out in the street, visiting friends), where a change in weather conditions and the amount of traffic can cause some anxiety and affect the level of confidence of a patient.

RELATING SUBJECTIVE VISUAL DISABILITY TO A MEASURE OF SEVERITY OF BINOCULAR VISUAL FIELD DEFECT

The results of this study indicate that subjective data can discriminate between patients with mild/moderate and advanced binocular visual field loss as defined in this study (see Methods). The best discriminators seems to be the second factor given by the factor structure in this study - that is, disability glare, adaptation to different levels of lighting and activities demanding functional peripheral vision. However, these subjective discriminators do not seem to be sensitive enough to detect differences between mild and moderate binocular field loss as defined in this study. Although patients with moderate damage may have some idea of increased difficulties with daily life activities in general, no difference is found between the two groups when performing any particular task. This suggests that patients may progress from the mild to the moderate stage of the visual field damage (as defined here) without noticing it in their daily routine. Normal subjects were not considered in this study as the purpose was to examine visual disability between groups with varying degrees of visual field loss.

Disability glare and lighting have separated mild/moderate from severe visual field loss in this study (Table 5). In everyday situations, glare disability is observed when driving at night against oncoming car's headlights or during sunny winter days, entering dark rooms, and when indoors with mirrored areas facing lighting sources. Although the results of this study in regard to disability glare and lighting cannot be directly compared with any other study, the work by Sherwood et al in a recent publication indicates that glaucoma patients perceive glare and night vision difficulty when compared with normals. ${ }^{17}$ In 1989 DenglerHarnes et al ${ }^{18}$ showed that forward light scatter exaggerates existing visual field loss in glaucoma patients. In 1992 Ochsner and Zrenner 
included some glaucoma patients in their glare sensitivity study, ${ }^{19}$ and suggested that changes in the visual acuity-luminance function accompanied with high glare sensitivity are most often due to pathological changes in neuronal circuity of the retina. They remark that sensitivity to glare is an unspecific ophthalmological symptom which can be caused by different anatomical structures, and although it can be related to optical and to cortical structures, it can also be due to defects in the neuronal mechanisms of the retina that control adaptation processes. ${ }^{19}$ Van den Berg found that visual acuity correlates rather weakly with the amount of scatter. ${ }^{20}$ Since the amount of scatter causes a considerable loss of visual function, the results of his study showed that for glare sensitive patients the standard Snellen visual acuity test gives a rather limited impression of visual handicap. Hoshino and $\mathrm{Mi}$ zokami found a significant correlation between glare sensitivity measured with the MillarNadler glare tester and central visual field damage in patients with early to middle stage glaucoma. ${ }^{21}$ Others studies ${ }^{9}$ have shown that objectively measured glare disability when taken together with other tests (especially contrast sensitivity and visual acuity) made a distinct contribution to the overall characterisation of visual function. The issue of dark adaptation in glaucoma was addressed by Glovinsky et al who found abnormal scotopic sensitivity in glaucoma patients when compared to normals. ${ }^{22}$ More research into the problems associated with intraocular light scatter, brightness acuity, and sensitivity and scotopic sensitivity in glaucoma is needed.

Our patients also had problems with vision in activities demanding functional peripheral vision, particularly when walking. With advancing glaucoma damage the number of subjective discriminators seems to increase (Table 4). These results reaffirm the conclusions of Mills and Drance ${ }^{11}$ who used the Esterman binocular test as an objective measure of visual function and compared the performance scores with the self reported disability in patients with severe visual field damage. They found a significant correlation between the Esterman test and responses to a short visual disability questionnaire, particularly in activities demanding peripheral vision-that is, questions on tripping over, bumping into people or objects, and following the line of print or finding the next line. ${ }^{11} \mathrm{~A}$ number of groups have recently demonstrated visual disability in glaucoma patients using a questionnaire. A study by Gutierrez et al showed that a steady decline characterised the relation between visual field loss and health related quality of life. ${ }^{23}$ Sherwood et $a l^{17}$ and Parrish et al ${ }^{24}$ found a correlation between increasing field loss and a reduction in activities of daily living.

Some other signs of deterioration in the quality of life of a given patient were found in this study by a loss in confidence when performing certain activities, especially outdoor mobility tasks (going out in the street).
Anxiety and loss of confidence seem to precede the stage of actual problems in performing the tasks.

As mentioned earlier the selection of questionnaire items in this study was based on several examples in the literature from a range of ophthalmic conditions including glaucoma. The summary measure of a single value used by some authors ${ }^{23}$ as a performance measure across a number of questions was therefore inappropriate in this situation because of the basis on which the questions were chosen.

The validity of the glaucoma specific subgroups of questions was shown by the significant correlation with severity of visual field loss (Table 4). This correlation was similar to the value published by Gutierrez et $a l^{23}$ in glaucoma patients. A high level of internal consistency was found in the questionnaire structure and the glaucoma specific subgroup of questions.

The influence of different forms of treatment (medical, laser, surgery) and in particular pupil diameter was not addressed in this study. Pilocarpine is known to cause a diffuse depression in the hill of vision as a result of pupil miosis. ${ }^{25}{ }^{26}$ Pupil enlargement may be associated with increased glare disability. ${ }^{27}$ Topical $\beta$ blockers can cause systemic side effects which may influence general well being and have a bearing on subjective responses. ${ }^{28} \mathrm{~A}$ recent study by Wang et al aimed at developing a research instrument for measuring the effect of glaucoma and its treatment on quality of life and functional status, ${ }^{29}$ concluded that the glaucoma disability index, a 31 item questionnaire showed high internal consistency and construct validity and is intended to be used to evaluate quality of life related to treatment. Sherwood et al found that glaucoma medication correlated with self reported glare disability and night vision problems. ${ }^{17}$ All of the aspects relating to treatment and side effects of therapy need further investigation. ${ }^{30}$ In our ongoing study we are also looking at the relation between other psychophysical tests of visual function and self reported visual impairment in glaucoma.

\section{Conclusion}

This study has shown that from a large set of questions on daily living activities, the responses of glaucoma patients can best be described by four different areas. These are outdoor mobility/navigation, glare and lighting and activities demanding functional peripheral vision, household tasks, and personal care. The results of this study also indicate that subjective data can discriminate between patients with mild/moderate and advanced binocular visual field loss, as defined in this study (see Methods). The signs of a reduction in quality of life were experienced in difficulty with adapting to glare and different levels of lighting and in activities demanding functional peripheral vision, particularly when walking (tripping over objects). A loss of confidence was apparent in patients when going out in the street, before the actual disability problems were noted. The validity of the glaucoma 
specific subgroup of questions was shown by a significant correlation with the severity of visual field loss. A high level of internal consistency was found in the questionnaire structure.

There is a clear need to find out more about visual disability in glaucoma. The results so far are challenging, particularly as experienced difficulties are a crucial outcome measure and quality of life indicator. As many reports indicate ${ }^{2172329}$ this aspect of care is essential to the treatment and management of the glaucoma patient. Further studies are needed if we are to address the questions and problems of visual disability in glaucoma.

1 Quigley H. Number of people with glaucoma worldwide. $\mathrm{Br}$

ff Ophthalmol 1996;80:389-93.
2 Zimmerman T, Karunaratne N, Fechtner R. Glaucoma: outcomeology (Parts I, II). F Glaucoma 1996;5:151-5.

3 Lee P. Outcomes and endpoints in glaucoma. $f$ Glaucoma 1996;5:295-7.

4 Hakkinen L. Vision in the elderly and its use in the social environment. Scand f Soc Med 1984;35:5-60.

5 Jette A, Branch L. Impairment and disability in the aged. $f$ Chronic Dis 1985;38:59-65.

6 Thomson J, Gibson J, Jagger C. The association between visual impairment and mortality in elderly people. Age Ageing 1989;18:83-8.

7 Bergman B, Sjostrand J. Vision and visual disability in the daily life of a representative population-sample aged 82 years. Acta Ophthalmol 1992;70:33-43.

8 Marx M, Werner P, Cohen-Mansfield J, et al. The relationship between low vision and performance of activities of daily living in nursing home residents. $\mathcal{F}$ Am Geriatr Soc 1992;40:1018-20.

9 Rubin GS, Roche KB, Prasada-Rao P, et al. Visual impairment and disability in older adults. Optom Vis Sci impairment and

10 Ross J, Bron A, Clarke D. Contrast sensitivity and visual disability in chronic-simple glaucoma. $\mathrm{Br} \mathcal{F}$ Ophthalmol 1984;68:821-7.

11 Mills R, Drance S. Esterman disability rating in severe glaucoma. Ophthalmology 1986;3:371-8.

12 Lundstrom M, Fregel G, Sjoblom A. Vision related daily life problems in patients waiting for a cataract extraction. $\mathrm{Br} F$ Ophthalmol 1994;78:608-11.
13 Cotton L. Visual and psychosocial characteristics of visually impaired older adults. [MSc by research.] Oxford: University of Oxford, 1995

14 Steinberg E, Tielsch J, Schein O, et al. The VF-14. An index of functional impairment in patients with cataract. Arch Ophthalmol 1994;112:630-8.

15 Mangione C, Phillips R, Seddon J, et al. Development of the "Activities of Daily Vision Scale". A measure of visual functional status. Medical Care 1992;30:1111-26.

16 Kline P. An easy guide to factor analysis. London: Routledge, 1994

17 Sherwood M, Garcia-Siekavizza A, Meltzer M, et al. Glaucoma's impact on quality of life and its relation to clinical indicators. Ophthalmology 1998;105:561-6.

18 Dengler-Harnes M, Wild J, Cole M, et al. The influence of forward light scatter on the visual field indices in glaucoma. Graefes Arch Clin Exp Ophthalmol 1990;228:326-31.

19 Ochsner H, Zrenner E. Vision and dazzle: II. The effect of increasing luminance on visual acuity of glare sensitive patients. Klin Monatsbl Augenheilkd 1992;200:110-17.

20 Van Den Berg T. Importance of pathological intraocular scatter for visual disability. Doc Ophthalmol 1985;61:327-

21 Hoshino M, Mizokami K. Glare disability in chronic glaucoma. Jpn f Clin Ophthalmol 1990;44:145-7.

22 Glovinsky Y, Quigley H, Drum B, et al. A whole-field scotopic retinal sensitivity test for the detection of early glaucoma damage. Arch Ophthalmol 1992;110:486-90.

23 Gutierrez P, Wilson M, Johnson C, et al. Influence of glaucomatous visual field loss on health-related quality of life. Arch Ophthalmol 1997;115:777-84.

24 Parrish II R, Gedde S, Scott I, et al. Visual function and quality of life among patients with glaucoma. Arch Ophthalmol 1997;115:1447-55

25 Lindenmuth K, Skuta G, Rabbani R, et al. Effects of pupillary constriction on automated perimetry in normal eyes. Ophthalmology 1989;96:1298-301.

26 Webster A, Luff A, Canning C, et al. The effect of pilocarpine on the glaucomatous visual field. Br F Ophthalmol 1993;77:721-5.

27 Masket S. Relationship between postoperative pupil size and disability glare. 7 Cataract Refract Surg 1992;18:506-7.

28 Diggory P, Franks AF. Glaucoma therapy may take your breath away. Age Ageing 1997;26:63-7.

29 Wang F, Javitt J, Rowe M, et al. Measuring the impact of glaucoma and its treatment on quality of life: the glaucoma disability index. Invest Ophthalmol Vis Sci 1996;37:S643.

30 Barber B, Strahlman E, Laibovitz R, et al. Validation of a questionnaire for comparing the tolerability of ophthalmic medications. Ophthalmology 1997;104:334-42. 